\title{
miR-217 inhibits osteogenic differentiation of rat bone marrow-derived mesenchymal stem cells by binding to Runx2
}

\author{
YU-LONG ZHU, SHUI WANG, DE-GANG DING, LIANG XU and HAI-TAO ZHU \\ Department of Orthopedics, Sheyang County People's Hospital, Yancheng, Jiangsu 224300, P.R. China
}

Received January 5, 2016; Accepted February 3, 2017

DOI: $10.3892 / \mathrm{mmr} .2017 .6349$

\begin{abstract}
The elucidation of the underlying molecular mechanisms regulating the osteogenic differentiation of bone marrow-derived mesenchymal stem cells (BMSCs) is of great importance in improving the treatment of bone-associated diseases. MicroRNAs (miRNAs) have been proven to regulate the osteogenic differentiation of BMSCs. The present study investigated the role of miR-217 in the osteogenic differentiation of rat BMSCs. It was observed that miR-217 expression levels were downregulated during the process of osteogenic differentiation. Subsequently, a dual-luciferase reporter gene assay demonstrated that miR-217 targets a putative binding site in the 3'-untranslated region of the runt related transcription factor 2 (Runx2) gene, which is a key transcription factor for osteogenesis. It was then demonstrated that overexpression of miR-217 attenuated the osteogenesis of BMSCs and downregulated the expression of Runx2, whereas inhibition of miR-217 promoted osteoblastic differentiation and upregulated Runx2 expression. Furthermore, the extracellular signal-regulated kinase (ERK) and p38 mitogen-activated protein kinase (p38 MAPK) signaling pathways were investigated during osteogenic induction, and the data indicated that miR-217 may exert a negative effect on the osteogenic differentiation of BMSCs through alteration of ERK and p38 MAPK phosphorylation. The present study therefore concluded that miR-217 functions as a negative regulator of BMSC osteogenic differentiation via the inhibition of Runx 2 expression, and the underlying molecular mechanisms may partially be attributed to mediation by the ERK and p38 MAPK signaling pathways.
\end{abstract}

\section{Introduction}

Bone homeostasis is maintained by the correct balance between bone formation, which is mediated by osteoblasts,

Correspondence to: Dr Hai-Tao Zhu, Department of Orthopedics, Sheyang County People's Hospital, 27 Fahong Street, Yancheng, Jiangsu 224300, P.R. China

E-mail: zhuhai_tao123@sina.com

Key words: microRNA-217, runt related transcription factor 2, osteogenic differentiation, bone marrow-derived mesenchymal stem cells and bone resorption mediated by osteoclasts, to regulate bone volume (1). Bone marrow-derived mesenchymal stem cells (BMSCs) are a population of self-renewing multipotent cells derived from bone marrow (2). Numerous studies have demonstrated that BMSCs may differentiate into osteoblasts, which makes them a suitable cell type for therapeutic use in bone repair and tissue engineering $(3,4)$. However, the regulation of these cellular pathways remains to be fully elucidated. Therefore, investigation of the regulatory mechanism of osteoblast differentiation is essential for the development of therapeutic strategies to treat bone loss (5).

MicroRNAs (miRNAs) are a class of endogenous non-coding single-stranded RNA molecules of 22 nucleotides in length, which function at the post-transcriptional level by negatively regulating gene expression via base pairing to complementary sites in the target mRNA 3' untranslated regions (UTR) (6). miRNAs have emerged as key post-transcriptional regulators of gene expression and are associated with a range of various physiological and pathological processes (7). It has previously been demonstrated that osteogenic induction and differentiation are regulated by post-transcriptional mechanisms, predominantly by temporally expressed miRNAs. Various miRNAs (miRs), including miR-30, miR-182 and miR-346 have been demonstrated to regulate the osteogenic differentiation of BMSCs (8-10). miR-30 inhibits osteoblast differentiation by targeting mothers against decapentaplegic homolog 1 and runt-related transcription factor 2 (Runx2) (8), miR-182 represses the osteogenesis of mesenchymal stem cells by targeting forkhead box O1 (9) and miR-346 regulates human osteogenic differentiation by targeting the Wnt/ $\beta$-catenin pathway (10). However, the specific role and function of miRNAs in osteogenic differentiation remains to be elucidated.

miR-217 was previously demonstrated to act as a tumor suppressor and is important in tumor cell proliferation and migration. The dysregulation of miR-217 has been reported in various tumor types including osteosarcoma (11), gastric cancer (12), pancreatic cancer (13) and clear cell renal cell carcinoma (14). However, studies remain to be conducted to investigate the role of miR-217 in the osteogenic differentiation of BMSCs.

In the present study, the expression of miR-217 during the osteogenic differentiation of BMSCs was first detected and it was observed that the expression of miR-217 was negatively associated with bone formation and osteogenic differentiation. 
Gain- and loss-of-function experiments subsequently demonstrated that miR-217 is a negative regulator of osteogenic differentiation in BMSCs. Luciferase assay and western blotting then verified that Runx2 is a target of miR-217 and the regulatory mechanisms may be mediated via extracellular signal-regulated kinase (ERK) and p38 mitogen-activated protein kinase (p38 MAPK) pathways.

\section{Materials and methods}

Isolation and culture of rat BMSCs. The Animal Research Ethics Committee of Sheyang County People's Hospital (Jiangsu, China) approved all animal experiments. Sprague-Dawley male rats (6 weeks old; $n=30$ ) weighing 200-250 g were provided by the Experimental Animal Center of Nanjing Medical University (Nanjing, China). They were housed under controlled conditions with a 12-h light/dark cycle at a temperature of $24 \pm 1^{\circ} \mathrm{C}$ and humidity of $50 \pm 5 \%$. The rats were allowed free access to standard rat chow and water. Rats were anesthetized via intraperitoneal injection of urethane (20\% in saline, $5 \mathrm{ml} / \mathrm{kg}$; Shanghai Qingxi Chemical Technology Co., Ltd., Shanghai, China). Bone marrow was flushed out from the tibiae and femurs of rats with sterilized PBS, and subsequently filtered through a strainer and suspended in Dulbecco's modified Eagle's medium (Sigma-Aldrich; Merck KGaA, Darmstadt, Germany), supplemented with $10 \%$ fetal bovine serum (Gibco; Thermo Fisher Scientific, Inc., Waltham, MA, USA), $10 \mathrm{U} / \mathrm{ml}$ penicillin and $100 \mu \mathrm{g} / \mathrm{ml}$ streptomycin (Hyclone; GE Healthcare Life Sciences, Logan, UT, USA). Cells were subsequently cultured at $37^{\circ} \mathrm{C}$ in an atmosphere containing $5 \% \mathrm{CO}_{2}$ for $24 \mathrm{~h}$. When cells reached $90 \%$ confluence, they were trypsinized and re-plated in $25 \mathrm{~cm}^{2}$ culture flasks. The resulting cultures were referred to as passage 0 (15). The passage 3 of BMSCs was used in the present study.

Osteogenesis-induced differentiation. The BMSCs were trypsinized and re-plated in 6-well plates at a concentration of $1 \times 10^{5}$ cells/well. When cells were $80-90 \%$ confluent, the medium was replaced by osteogenic induction medium containing $100 \mathrm{nM}$ dexamethasone, $50 \mathrm{mg} / \mathrm{ml}$ ascorbate, $10 \mathrm{mM} \beta$-glycerophosphate and $25 \mathrm{ng} / \mathrm{ml}$ recombinant human bone morphogenetic protein 2 precursor (Bmp2; Sigma-Aldrich; Merck KGaA). The medium was replaced every 3 days for 3 weeks.

Alizarin red staining. Alizarin red staining was used to detect the calcium deposits. A total of $1 \times 10^{5}$ cells were washed twice with PBS after 21 days, following fixation with paraformaldehyde for $30 \mathrm{~min}$ at $4^{\circ} \mathrm{C}$ and then washed with double distilled water $\left(\mathrm{ddH}_{2} \mathrm{O}\right)$ three times. The fixed cells were subsequently stained with $0.1 \%$ alizarin red (Sigma-Aldrich; Merck KGaA) solution for $30 \mathrm{~min}$ at room temperature. The cells were then washed twice with PBS and observed under a microscope (Olympus Corporation, Tokyo, Japan; x50 magnification).

Reverse transcription-quantitative polymerase chain reaction $(q P C R)$. Total RNA from the BMSCs was extracted using an RNeasy Mini kit (Qiagen China Co., Ltd., Shanghai, China), according to the manufacturer's protocol. The mRNA was reverse transcribed to cDNA using the PrimeScript 1st strand cDNA synthesis kit (Takara Biotechnology Co., Ltd., Dalian, China). The PCR amplification was conducted in a total volume of $20 \mu \mathrm{l}$, containing $0.4 \mu \mathrm{l}$ each primer, $10 \mathrm{ml} \mathrm{SYBR}{ }^{\circledR}$ Green qPCR Master Mix (Takara Biotechnology Co., Ltd.), $2 \mu \mathrm{l}$ cDNA and $7.2 \mu 1 \mathrm{H}_{2} \mathrm{O}$. Primer sequences are presented in Table I. Generation of standard curves and qPCR were carried out using an ABI7500 Real-Time PCR instrument (Applied Biosystems; Thermo Fisher Scientific, Inc.). The thermocycling conditions were $95^{\circ} \mathrm{C}$ for $10 \mathrm{~min}$ followed by 40 cycles of $95^{\circ} \mathrm{C}$ for $5 \mathrm{sec}$ and $60^{\circ} \mathrm{C}$ for $30 \mathrm{sec}$. The expression of the target gene was normalized to the geometric mean of the GAPDH housekeeping gene.

Total miRNA was extracted using the miRNeasy Mini Kit (Qiagen China Co., Ltd.) and reverse transcribed to form cDNA by TaqMan miRNA reverse transcription kit (Applied Biosystems, Thermo Fisher Scientific Inc.). Their primer sequences are presented in Table I. The expression levels of miRNA were measured using the TaqMan miRNA Assay kit (Applied Biosystems, Thermo Fisher Scientific Inc.) and normalized to U6 snRNA transcript levels. All relative gene expression levels were analyzed using the $2^{-\Delta \Delta C q}$ formula (16). Each sample was examined in triplicate and a no-template control was included for each amplification.

Transfection of miRNA mimics and inhibitor. miR-217 mimics, anti-miR-217 oligos and a scramble negative oligo control were purchased from Applied Biosystems (Rockville, MD, USA). Diethylpyrocarbonate-treated water was used to dissolve the oligonucleotides. A total of $50 \mathrm{nM}$ miRNA precursor or $10 \mathrm{nM}$ antisense oligos were transfected into cells using Lipofectamine ${ }^{\circledR} 2000$ (Invitrogen, Thermo Fisher Scientific, Inc.) according to the manufacturer's protocol. The cells were harvested after $48 \mathrm{~h}$. For long-term detection, mimics, inhibitors and negative control were repeatedly transfected every 3 days. miR-217 mimic sequences were 5'-GUC AGUCAAGGACUACGTCAU-3'; miR-217 inhibitor sequence was 5'-AUGACGUAGUCCUUGACUGAC-3' and the negative control sequence was 5'-CAGUACUUUUGUGTAGUA CAA-3'.

Western blotting. Total protein was extracted from BMSCs using lysis buffer $(50 \mathrm{mmol} / \mathrm{l}$ Tris, $150 \mathrm{mmol} / \mathrm{l} \mathrm{NaCl}, 1 \%$ Triton X-100, 1\% deoxycholic phenylmethylsulfonyl fluoride, $1 \mu \mathrm{g} / \mathrm{ml}$ aprotinin, $5.0 \mathrm{mmol}$ sodium pyrophosphate, $1.0 \mathrm{~g} / \mathrm{ml}$ leupeptin, $0.1 \mathrm{mmol}$ phenylmethylsulfonyl fluoride and $1 \mathrm{mmol} / \mathrm{l}$ dithiothreitol) on ice for $30 \mathrm{~min}$, and protein concentrations were quantified using the Pierce BCA Protein Assay kit (Thermo Fisher Scientific, Inc.). Proteins (25 $\mu \mathrm{g})$ were subsequently separated using SDS-PAGE on a $12 \%$ gel and electrophoretically transferred onto polyvinylidene difluoride membranes. The membranes were blocked with 5\% fat-free milk in TBS Tween-20 for $1 \mathrm{~h}$ at room temperature and then incubated with primary antibodies at $4^{\circ} \mathrm{C}$ overnight. Blots were incubated with horseradish peroxidase-conjugated rabbit anti-rat secondary antibody (catalog no. ab6734; 1:5,000; Abcam, Cambridge, UK) for $1 \mathrm{~h}$ at room temperature. The immunoreactive bands were detected by enhanced chemiluminescence detection reagents (GE Healthcare Life Sciences, Chalfont, UK). 
Table I. Oligonucleotide primer sequences and product sizes for reverse transcription-quantitative polymerase chain reaction.

\begin{tabular}{llll}
\hline Gene & \multicolumn{1}{c}{ Forward primer sequence } & Reverse primer sequence & Size $(\mathrm{bp})$ \\
\hline Runx2 & GCCACTTACCACAGAGCTATTA & GGCGGTCAGAGAACAAACTA & 106 \\
Alp & CATGTTCCTGGGAGATGGTAT & GTGTTGTACGTCTTGGAGAGA & 144 \\
Bmp2 & TGTGAGGATTAGCAGGTCTTT & TTGTGGAGTGGATGTCCTTTAC & 105 \\
OPN & CCCATCTCAGAAGCAGAATCTT & GTCATGGCTTTCATTGGAGTT & 109 \\
OC & TGACTGCATTCTGCCTCTC & CGGAGTCTATTCACCACCTTA & 109 \\
GAPDH & ACTCCCATTCTTCCACCTTT & CCCTGTTGCTGTAGCCATATT & 105
\end{tabular}

Runx2, runt related transcription factor 2; ALP, alkaline phosphatase; Bmp2, bone morphogenetic protein 2 precursor; OPN, osteopontin; OC, osteocalcin.

Antibodies. Anti-Runx2 (catalog no. ab23981; 1:1,000) and anti-Bmp2 (catalog no. ab14933; 1:1,000) were obtained from Abcam. Anti-alkaline phosphatase (ALP; catalog no. sc271431; 1:1,000), anti-osteocalcin (OC; catalog no. sc18319; 1:1,000), anti-osteopontin (OPN; catalog no. sc20788; 1:1,000), anti-phosphorylated (p)-ERK (catalog no. sc7383; 1:500), anti-ERK (catalog no. sc292838; 1:500), anti-p-p38 MAPK (catalog no. sc101758; 1:500) and anti-p38 MAPK (catalog no. sc7972; 1:500) were obtained from Santa Cruz Biotechnology Inc., Dallas, TX, USA. Anti-GAPDH (catalog no. TA309157; 1:1,000) was purchased from ZSGB-Bio (Beijing, China).

Luciferase assay. The full length Runx2 3'-UTR (Shanghai GenePharma Co., Ltd., Shanghai, China) and miR-217 binding sites were cloned into pGL3 vectors (Huada Genomics, Shenzheng, China). HEK293T cells were purchased from American Type Culture Collection (Teddington, UK) and cultured with high-glucose Dulbecco's modified Eagle's medium (DMEM; HyClone, GE Healthcare Life Sciences) supplemented with penicillin-streptomycin, fetal bovine serum and $0.25 \%$ trypsin-EDTA (Gibco; Thermo Fisher Scientific, Inc.). HEK293T cells were transfected with miR-217 mimic, or control at a final concentration of $50 \mathrm{nM}$ using Lipofectamine ${ }^{\circledR}$ 2000. The following day, cells were transfected with $200 \mathrm{ng}$ 3'-UTR plasmids along with Renilla luciferase plasmid (Promega Corporation, Madison, WI, USA) using XtremeGENE 9 (Roche Diagnostics, Basel, Switzerland), according to the manufacturer's protocol. Cell were harvested and lysed after $48 \mathrm{~h}$. Luciferase activity was measured using dual-luciferase assay kit (Promega Corporation) at a wavelength of $480 \mathrm{~nm}$. Renilla luciferase activity at $560 \mathrm{~nm}$ was used for normalization. The experiments were performed independently three times.

Statistical analysis. Data are expressed as the mean \pm standard deviation. Univariate comparison of means was evaluated using an unpaired Student's t-test. A value of $\mathrm{P}<0.05$ was considered to indicate a statistically significant difference. All statistical analysis was performed using SPSS 17.0 software (SPSS Inc., Chicago, IL, USA).

Bioinformatics predictions. To predict the target genes of miR-217 during the osteogenic differentiation, the present study selected two miRNA target prediction databases: TargetScan (http://www.targetscan.org) and miRanda (http://www.microrna.org).

\section{Results}

Osteogenic differentiation of rat BMSCs. BMSCs were treated with standard osteogenic-induction medium in order to induce osteogenic differentiation. This was detected by increased mRNA (data not shown) and protein expression levels of osteogenic-associated genes, including Runx2, ALP, OC, OPN, and Bmp2, at days 14 and 21 (Fig. 1A). Alizarin red staining for matrix mineralization confirmed the osteoblast phenotype (Fig. 1B). Increased expression of osteogenic-associated genes and the observed osteoblast phenotype were in accordance with previous reports describing rat BMSCs differentiation $(17,18)$.

miR-217 directly represses Runx2 expression in BMSCs. The expression of miR-217 was significantly decreased in a time-dependent manner during osteogenic differentiation of BMSCs (Fig. 2A). Bioinformatic analyses were then conducted to predict the target genes of miR-217 using Targetscan (www.targetscan.org) and miRanda (www. microrna.org) (19). The analysis of the 3'-UTR of the Runx2 mRNA revealed potential binding sites for miR-217, which suggested the existence of a regulatory association between miR-217 and Runx2 (Fig. 2B). To assess whether Runx2 is a direct target of $\mathrm{miR}-217$, the dual-luciferase reporter gene assay was performed. The results demonstrated that upregulation of miR-217 may decrease the luciferase activity of the WT-Runx2-3'-UTRs of Runx2 and the luciferase activity was observed to be $\sim 40 \%$ compared with negative control. Furthermore, no significant effect was observed on the Mut-Runx2-3'-UTRs (Fig. 2C). These results suggested that miR-217 mimics may inhibit Runx 2 expression via a pairing with the Runx2 3'-UTR binding site.

miR-217 inhibits osteogenic differentiation. To investigate the role of miR-217 in osteogenic differentiation, BMSCs were induced to differentiate into osteoblasts following transfection with miR-217 mimics or anti-miR-217 (Fig. 3A). Inhibiting miR-217 dramatically enhanced osteoblastic differentiation, which was indicated by enhanced in vitro 
A

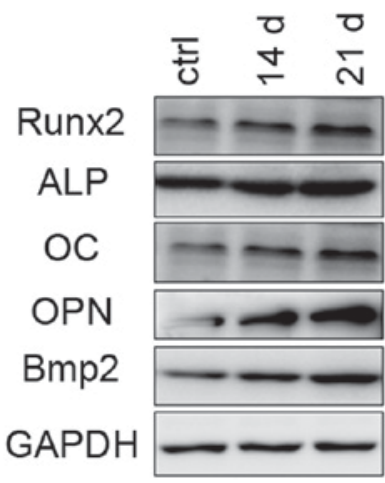

\section{B $\quad 0 d$}

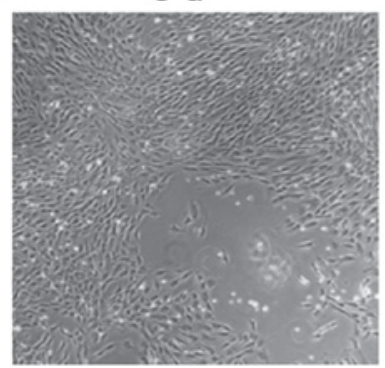

$14 \mathrm{~d}$

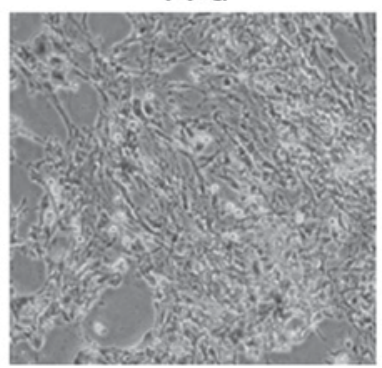

$21 d$

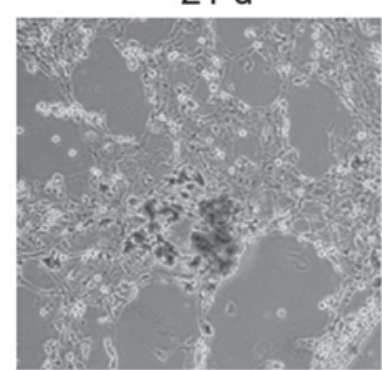

Figure 1. Osteogenic differentiation was induced by osteogenic-induction medium. (A) Protein expression of Runx2, ALP, OC, OPN and Bmp2 during osteogenic differentiation. (B) Alizarin red staining images are presented at 0, 14 and 21 days. ALP, alkaline phosphatase; OC, osteocalcin; OPN, osteopontin; Bmp2, bone morphogenetic protein 2 precursor; ctrl, control; Runx2, runt related transcription factor 2.

A

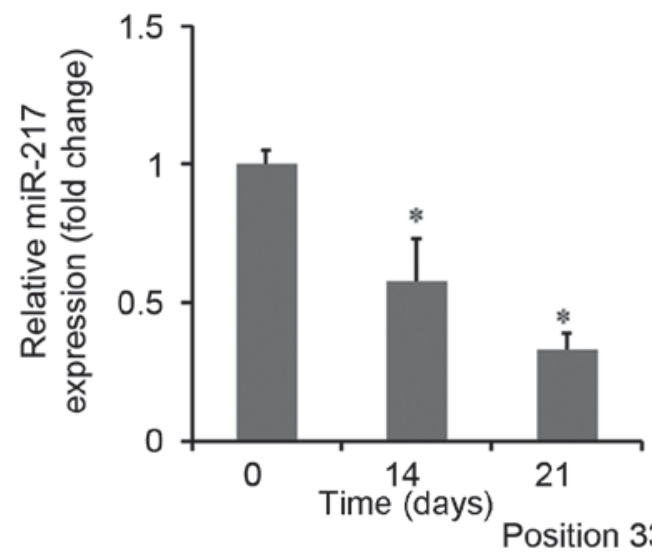

B

Runx2 3' UTR

Position 3364-3371

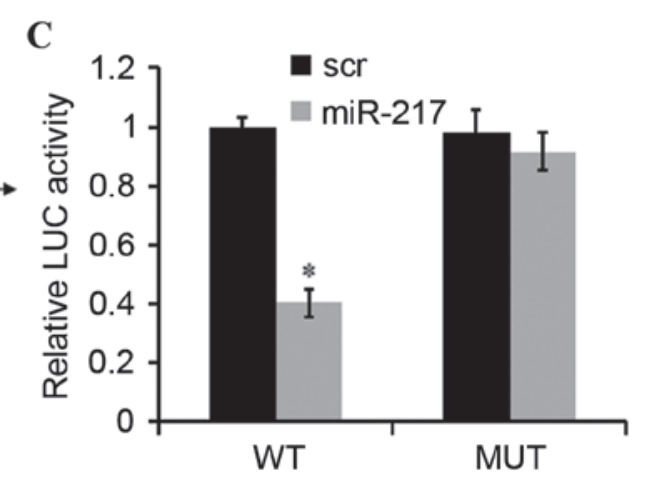

Figure 2. Runx2 is a target of miR-217. (A) Expression of miR-217 in osteogenic differentiation of BMSCs at days 0, 14 and 21. (B) Schematic illustration of the predicted miR-217-binding sites in the 3'-UTR of Runx2; (C) BMSCs were co-transfected with luciferase reporter plasmids containing WT or Mut miR-217 target sites in the Runx2. Data are expressed as the mean \pm standard deviation. " $\mathrm{P}<0.05$ vs. 0 days. BMSCs, bone marrow-derived mesenchymal stem cells; Runx2, runt related transcription factor 2; UTR, untranslated region; WT, wild-type; Mut, mutated; miR, microRNA.

matrix mineralization visualized by alizarin red staining (Fig. 3B). By contrast, matrix mineralization was observed to be reduced in miR-217 mimics-transfected BMSCs and cells transfected with negative control (Fig. 3B). In addition, the expression levels of the osteoblast-specific genes Runx2, ALP, OC, OPN and Bmp2 appeared to be increased by inhibition of miR-217, and decreased in the mimic and control groups (Fig. 4A). 

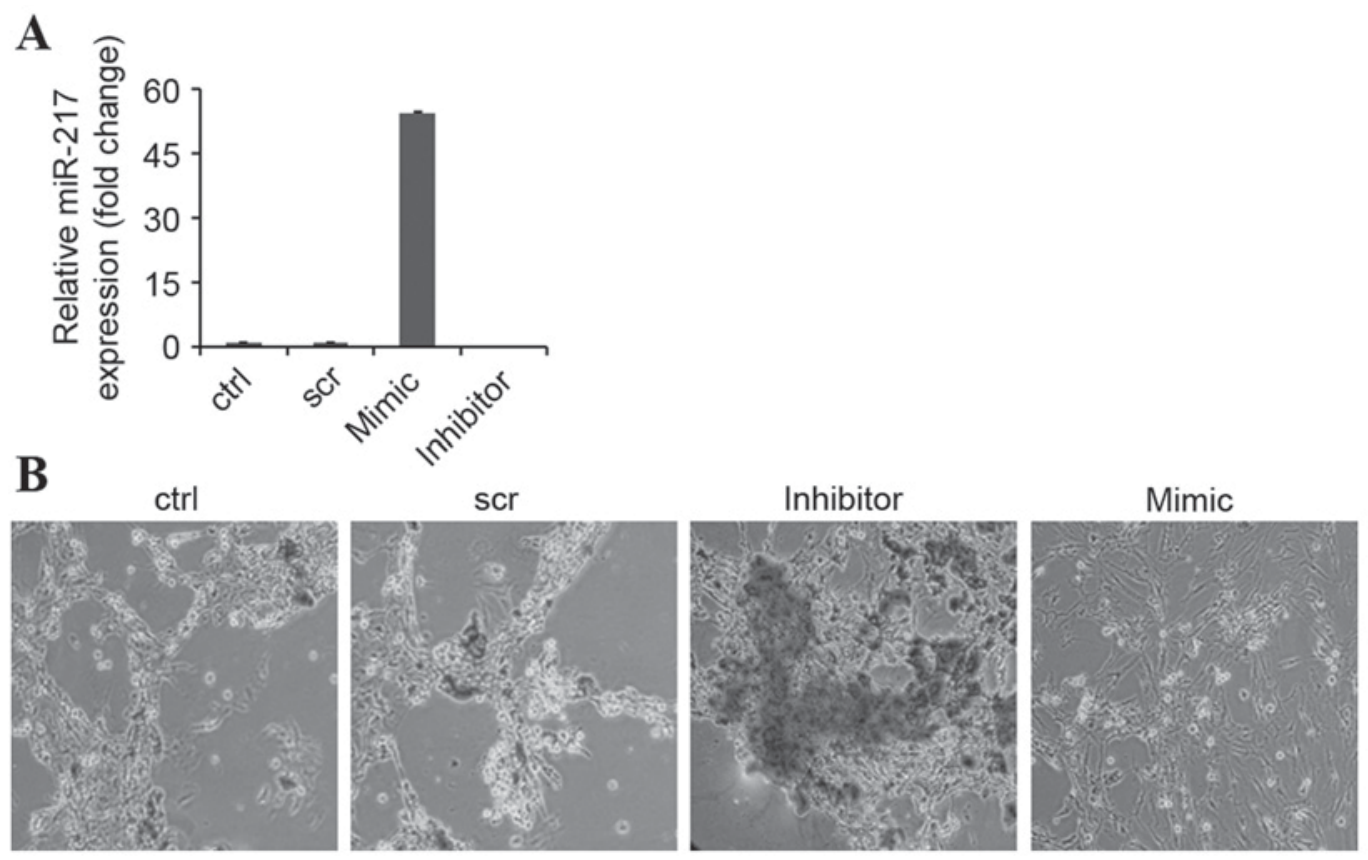

Figure 3. Effects of miR-217 on the osteogenic differentiation of BMSCs. (A) Expression of miR-217 in BMSCs following treatment with scr negative control, miR-217 inhibitor or miR-217 mimics. (B) Alizarin red staining images presented at 14 days. BMSCs, bone marrow-derived mesenchymal stem cells; scr, scrambled; ctrl, control; miRNA, microRNA.

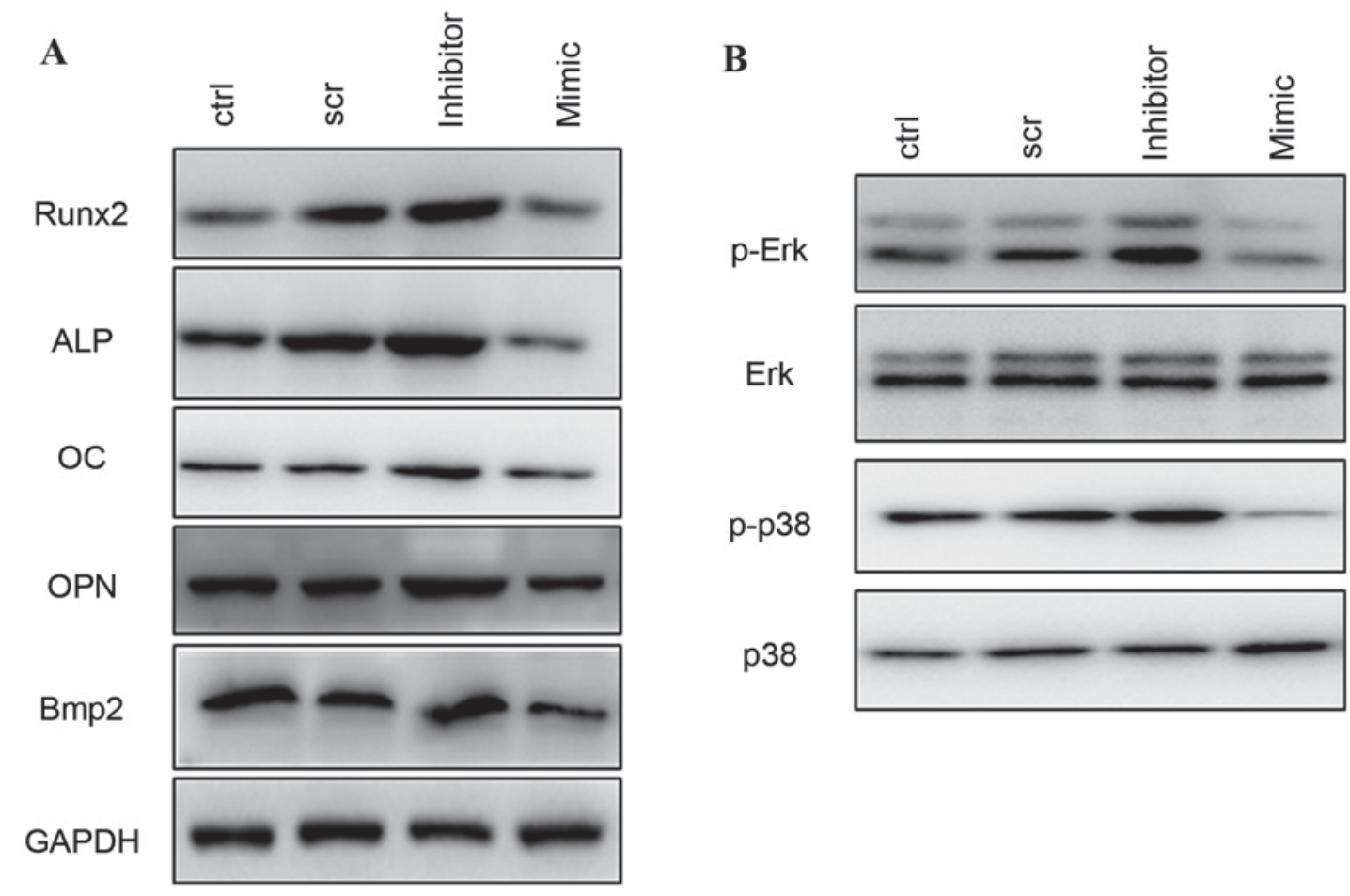

Figure 4. Western blotting of osteogenic differentiation-associated proteins. (A) Protein expression levels of Runx2, ALP, OC, OPN, and Bmp2 following treatment with scr, miR-217 inhibitor or miR-217 mimics. (B) Phosphorylation levels of ERK and p38 MAPK following transfection with scr, miR-217 inhibitor or mimics. ERK, extracellular signal-regulated kinase; MAPK, mitogen-activated protein kinase; scr, scrambled; ctrl, control; miRNA, microRNA; ALP, alkaline phosphatase; OC, osteocalcin; OPN, osteopontin; Bmp2, bone morphogenetic protein 2 precursor, ctrl, control; Runx2, runt related transcription factor 2.

Silencing of miR-217 increases ERK and p38 activation. To investigate the mechanism involved in the regulation of osteogenic differentiation by miR-217, the present study detected the phosphorylation level of ERK and p38 MAPK in response to osteogenic stimulation. Following transfection with negative control, miR-217 inhibitor or mimics for $48 \mathrm{~h}$, it was observed that inhibition of miR-217 markedly increased phosphorylation of ERK1/2 and p38 MAPK, whereas overexpression of miR-217 decreased phosphorylated ERK1/2 and p38 MAPK during osteogenic differentiation (Fig. 4B). 


\section{Discussion}

BMSCs are multipotent cells that, when under the appropriate conditions, differentiate into osteoblastic cells. The present study identified miR-217 as a negative regulator of BMSC osteogenic differentiation via inhibition of the expression of Runx2, and the underlying molecular mechanism may be mediated in part by the ERK and p38 MAPK signaling pathways.

Deregulation of miRNA mediated mechanisms is pathologically associated with osteoporosis and other bone-associated diseases $(20,21)$. Increasing evidence in recent years has demonstrated that miRNA are important regulators in osteogenic differentiation of BMSCs (22-25). The present study demonstrated that miR-217 is downregulated during osteogenic differentiation. Inhibition of miR-217 function promoted osteogenic differentiation of BMSCs, whereas overexpression of miR-217 attenuated it. These findings suggest that miR-217 is important in bone formation by negatively regulating osteogenic differentiation.

To further investigate the underlying molecular mechanism of miR-217 in regulating osteoblast differentiation of BMSCs, the present study searched for potential target genes with an established or potential function in osteogenesis. Notably, a match between the miR-217 seed region and the 3' UTR of Runx2 was identified. It was subsequently demonstrated that miR-217 overexpression results in downregulation of Runx2, whereas inhibition of miR-217 reverses this effect, suggesting that Runx 2 is regulated by miR-217 during osteogenic differentiation. Runx2 is a key transcription factor associated with osteogenic differentiation. Targeted disruption of Runx 2 in mice results in the maturational arrest of osteoblasts and a complete lack of mineralized bone $(26,27)$. The epigenetic functions of Runx 2 regulate expression of bone-associated genes (28), which accounts for the observed miR-217-induced downregulation of ALP, OC, OPN, and Bmp2. miR-217 was a primary inhibitor of osteoblastic differentiation by directly targeting Runx2.

Experimental evidence suggests that the MAPK signaling pathway is essential during the initiation stage of osteogenic differentiation (29). Critical members of the MAPKs include ERK1/2, which is an essential molecule for mechanotransduction (30). Runx 2 is phosphorylated and activated by the ERK1/2 signaling pathways (31). However, the association between miR-217 and the ERK/p38 pathways remains to be elucidated. Therefore, the present study further detected the phosphorylation of ERK and p38 MAPK in BMSCs. Upregulation of miR-217 resulted in a decrease in phosphorylation of ERK and p38 MAPK, suggesting that miR-217 may negatively regulate osteogenic differentiation via the MAPK/ERK signaling pathway. The present study therefore hypothesized that miR-217 may negatively regulate osteogenic differentiation in BMSCs, via alteration of the phosphorylation of ERK and p38 MAPK.

In conclusion, the results demonstrated that the expression of miR-217 was decreased during osteogenic differentiation of rat BMSCs and miR-217 inhibited osteogenic differentiation via directly suppressing Runx 2 expression and thereby inhibiting expression of osteoblast-associated genes. Furthermore, the possible mechanisms may partly be mediated by the ERK and p38 MAPK pathways. These observations are novel in describing the role of miRNAs in osteoblast differentiation.

\section{References}

1. Corral DA, Amling M, Priemel M, Loyer E, Fuchs S, Ducy P, Baron R and Karsenty G: Dissociation between bone resorption and bone formation in osteopenic transgenic mice. Proc Natl Acad Sci USA 95: 13835-13840, 1998.

2. Charbord P: Bone marrow mesenchymal stem cells: Historical overview and concepts. Hum Gene Ther 21: 1045-1056, 2010

3. Sacchetti B, Funari A, Michienzi S, Di Cesare S, Piersanti S, Saggio I, Tagliafico E, Ferrari S, Robey PG, Riminucci M and Bianco P: Self-renewing osteoprogenitors in bone marrow sinusoids can organize a hematopoietic microenvironment. Cell 131: 324-336, 2007.

4. Mathews S, Bhonde R, Gupta PK and Totey S: Extracellular matrix protein mediated regulation of the osteoblast differentiation of bone marrow derived human mesenchymal stem cells. Differentiation 84: 185-192, 2012.

5. Khosla S, Westendorf JJ and Oursler MJ: Building bone to reverse osteoporosis and repair fractures. J Clin Invest 118: 421-428, 2008.

6. Ghildiyal $M$ and Zamore PD: Small silencing RNAs: An expanding universe. Nat Rev Genet 10: 94-108, 2009.

7. Ha M and Kim VN: Regulation of microRNA biogenesis. Nat Rev Mol Cell Biol 15: 509-524, 2014.

8. Wu T, Zhou H, Hong Y, Li J, Jiang X and Huang H: miR-30 family members negatively regulate osteoblast differentiation. J Biol Chem 287: 7503-7511, 2012.

9. Kim KM, Park SJ, Jung SH, Kim EJ, Jogeswar G, Ajita J, Rhee Y, $\mathrm{Kim} \mathrm{CH}$ and Lim SK: miR-182 is a negative regulator of osteoblast proliferation, differentiation, and skeletogenesis through targeting FoxO1. J Bone Miner Res 27: 1669-1679, 2012.

10. Wang Q, Cai J, Cai XH and Chen L: miR-346 regulates osteogenic differentiation of human bone marrow-derived mesenchymal stem cells by targeting the Wnt/ $/$-catenin pathway. PLoS One 8: e72266, 2013.

11. Wei R, Deng Z and Su J: miR-217 targeting Wnt5a in osteosarcoma functions as a potential tumor suppressor. Biomed Pharmacother 72: 158-164, 2015.

12. Chen DL, Zhang DS, Lu YX, Chen LZ, Zeng ZL, He MM, Wang FH, Li YH, Zhang HZ, Pelicano H, et al: microRNA-217 inhibits tumor progression and metastasis by downregulating EZH2 and predicts favorable prognosis in gastric cancer. Oncotarget 6: 10868-10879, 2015.

13. Zhao WG, Yu SN, Lu ZH, Ma YH, Gu YM and Chen J: The miR-217 microRNA functions as a potential tumor suppressor in pancreatic ductal adenocarcinoma by targeting KRAS Carcinogenesis 31: 1726-1733, 2010.

14. Li H, Zhao J, Zhang JW, Huang QY, Huang JZ, Chi LS, Tang HJ, Liu GQ, Zhu DJ and Ma WM: MicroRNA-217, down-regulated in clear cell renal cell carcinoma and associated with lower survival, suppresses cell proliferation and migration. Neoplasma 60: 511-515, 2013.

15. Pan GZ, Yang Y, Zhang J, Liu W, Wang GY, Zhang YC, Yang Q, Zhai FX, Tai Y, Liu JR, et al: Bone marrow mesenchymal stem cells ameliorate hepatic ischemia/reperfusion injuries via inactivation of the MEK/ERK signaling pathway in rats. J Surg Res 178: 935-948, 2012.

16. Livak KJ and Schmittgen TD: Analysis of relative gene expression data using real-time quantitative PCR and the 2(-Delta Delta C(T)) Method. Methods 25: 402-408, 2001.

17. Qu B, Xia X, Wu HH, Tu CQ and Pan XM: PDGF-regulated miRNA-138 inhibits the osteogenic differentiation of mesenchymal stem cells. Biochem Biophys Res Commun 448: 241-247, 2014.

18. Xu L, Liu Y, Hou Y, Wang K, Wong Y, Lin S and Li G: U0126 promotes osteogenesis of rat bone-marrow-derived mesenchymal stem cells by activating BMP/Smad signaling pathway. Cell Tissue Res 359: 537-545, 2015.

19. Lewis BP, Shih IH, Jones-R hoades MW, Bartel DP and Burge CB: Prediction of mammalian microRNA targets. Cell 115: 787-798, 2003.

20. Krzeszinski JY, Wei W, Huynh H, Jin Z, Wang X, Chang TC, Xie XJ, He L, Mangala LS, Lopez-Berestein G, et al: miR-34a blocks osteoporosis and bone metastasis by inhibiting osteoclastogenesis and Tgif2. Nature 512: 431-435, 2014. 
21. Garnero P: New developments in biological markers of bone metabolism in osteoporosis. Bone 66: 46-55, 2014.

22. Qadir AS, Um S, Lee H, Baek K, Seo BM, Lee G, Kim GS, Woo KM, Ryoo HM and Baek JH: miR-124 negatively regulates osteogenic differentiation and in vivo bone formation of mesenchymal stem cells. J Cell Biochem 116: 730-742, 2015.

23. Huszar JM and Payne CJ: MIR146A inhibits JMJD3 expression and osteogenic differentiation in human mesenchymal stem cells. FEBS Lett 588: 1850-1856, 2014.

24. Huang K, Fu J, Zhou W, Li W, Dong S, Yu S, Hu Z, Wang H and Xie Z: MicroRNA-125b regulates osteogenic differentiation of mesenchymal stem cells by targeting $\operatorname{Cbf} \beta$ in vitro. Biochimie 102: 47-55, 2014.

25. Deng Y, Wu S, Zhou H, Bi X, Wang Y, Hu Y, Gu P and Fan X: Effects of a miR-31, Runx2, and Satb2 regulatory loop on the osteogenic differentiation of bone mesenchymal stem cells. Stem Cells Dev 22: 2278-2286, 2013.

26. Komori T, Yagi H, Nomura S, Yamaguchi A, Sasaki K, Deguchi K, Shimizu Y, Bronson RT, Gao YH, Inada M, et al Targeted disruption of Cbfal results in a complete lack of bone formation owing to maturational arrest of osteoblasts. Cell 89 755-764, 1997.
27. Yoshida CA, Furuichi T, Fujita T, Fukuyama R, Kanatani N, Kobayashi S, Satake M, Takada K and Komori T: Core-binding factor beta interacts with Runx2 and is required for skeletal development. Nat Genet 32: 633-638, 2002.

28. Lian JB and Stein GS: Runx2/Cbfa1: A multifunctional regulator of bone formation. Curr Pharm Des 9: 2677-2685, 2003

29. Lai CF, Chaudhary L, Fausto A, Halstead LR, Ory DS, Avioli LV and Cheng SL: Erk is essential for growth, differentiation, integrin expression, and cell function in human osteoblastic cells J Biol Chem 276: 14443-14450, 2001.

30. Simmons CA, Matlis S, Thornton AJ, Chen S, Wang CY and Mooney DJ: Cyclic strain enhances matrix mineralization by adult human mesenchymal stem cells via the extracellular signal-regulated kinase (ERK1/2) signaling pathway. J Biomech 36: 1087-1096, 2003

31. Xiao G, Jiang D, Thomas P, Benson MD, Guan K, Karsenty G and Franceschi RT: MAPK pathways activate and phosphorylate the osteoblast-specific transcription factor, Cbfa1. J Biol Chem 275: 4453-4459, 2000. 